\title{
Correctness of belief propagation in Gaussian graphical models of arbitrary topology
}

Yair Weiss

Computer Science Division 485 Soda Hall

UC Berkeley

Berkeley, CA 94720-1776 yweiss@cs.berkeley.edu
William T. Freeman

MERL, Mitsubishi Electric Research Labs. 201 Broadway

Cambridge, MA 02139

freeman@merl.com

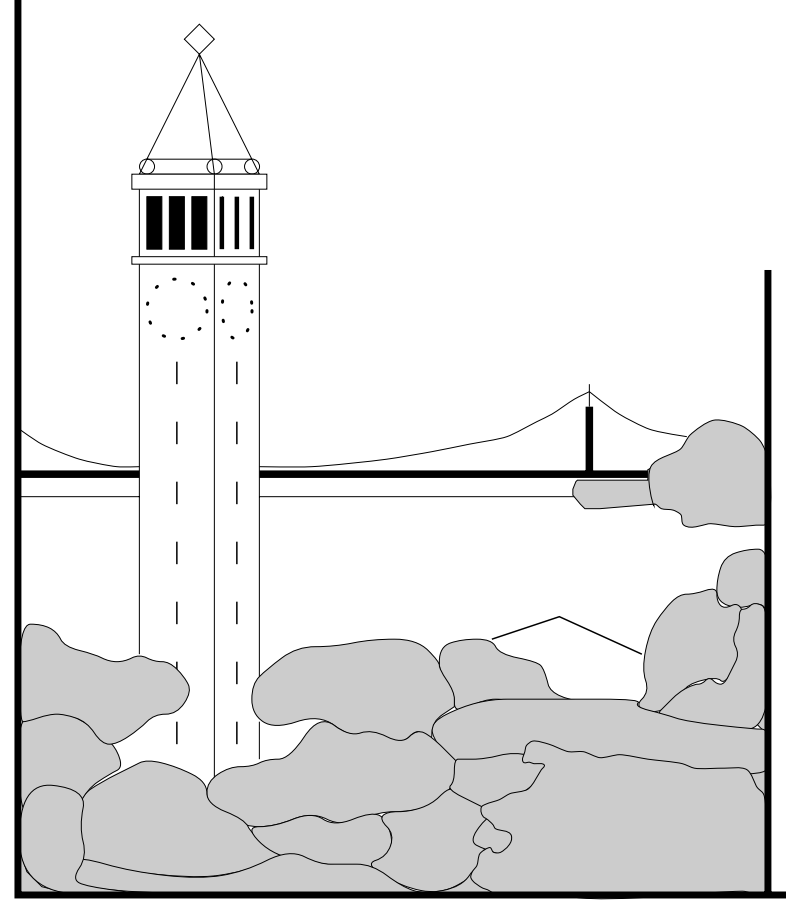

Report No. UCB/CSD-99-1046

June 1999

Computer Science Division (EECS)

University of California

Berkeley, California 94720 


\begin{abstract}
Graphical models, such as Bayesian networks and Markov Random Fields represent statistical dependencies of variables by a graph. Local "belief propagation" rules of the sort proposed by Pearl [18] are guaranteed to converge to the correct posterior probabilities in singly connected graphical models. Recently, a number of researchers have empirically demonstrated good performance of "loopy belief propagation"-using these same rules on graphs with loops. Perhaps the most dramatic instance is the near Shannonlimit performance of "Turbo codes", whose decoding algorithm is equivalent to loopy belief propagation.
\end{abstract}

Except for the case of graphs with a single loop, there has been little theoretical understanding of the performance of loopy propagation. Here we analyze belief propagation in networks with arbitrary topologies when the nodes in the graph describe jointly Gaussian random variables. We give an analytical formula relating the true posterior probabilities with those calculated using loopy propagation. We give sufficient conditions for convergence and show that when belief propagation converges it gives the correct posterior means for all graph topologies, not just networks with a single loop.

The related "max-product" belief propagation algorithm finds the maximum posterior probability estimate for singly connected networks. We show that, even for non-Gaussian probability distributions, the convergence points of the max-product algorithm in loopy networks are at least local maxima of the posterior probability.

These results motivate using the powerful belief propagation algorithm in a broader class of networks, and help clarify the empirical performance results.

YW is supported by MURI-ARO-DAAH04-96-1-0341 
Problems involving probabilistic belief propagation arise in a wide variety of applications, including error correcting codes, speech recognition and medical diagnosis. Typically, a probability distribution is assumed over a set of variables and the task is to infer the values of the unobserved variables given the observed ones. The assumed probability distribution is described using a graphical model [13] — the qualitative aspects of the distribution are specified by a graph structure. The graph may either be directed as in a Bayesian network $[18,11]$ or undirected as in a Markov Random Field [18, 10]. Different communities tend to prefer different graph formalisms (see [19] for a recent review) — directed graphs are more common in AI, medical diagnosis and statistics while undirected graphs are more common in image processing, statistical physics and error correcting codes. In this paper we use the undirected graph formulation because one can always perform inference on a directed graph by converting it to an equivalent undirected graph.

If the graph is singly connected (i.e. there is only one path between any two given nodes) then there exist efficient local message-passing schemes to calculate the posterior probability of an unobserved variable given the observed variables. Pearl (1988) derived such a scheme for singly connected Bayesian networks and showed that this "belief propagation" algorithm is guaranteed to converge to the correct posterior probabilities (or "beliefs"). However, as Pearl noted, the same algorithm will not give the correct beliefs for multiply connected networks:

When loops are present, the network is no longer singly connected and local propagation schemes will invariably run into trouble... If we ignore the existence of loops and permit the nodes to continue communicating with each other as if the network were singly connected, messages may circulate indefinitely around the loops and the process may not converge to a stable equilibrium ... Such oscillations do not normally occur in probabilistic networks ... which tend to bring all messages to some stable equilibrium as time goes on. However, this asymptotic equilibrium is not coherent, in the sense that it does not represent the posterior probabilities of all nodes of the network (Pearl 1988, p. 195)

Despite these reservations, Pearl advocated the use of belief propagation in loopy networks as an approximation scheme (J. Pearl, personal communication) and one of the exercises in [18] investigates the quality of the approximation when it is applied to a particular loopy belief network.

Several groups have recently reported excellent experimental results by using this approximation scheme - by running algorithms equivalent to Pearl's algorithm on networks with loops $[8,17,7]$. Perhaps the most dramatic instance of this performance is in an error correcting code scheme known as "Turbo codes" [3]. These codes have been described as "the most exciting and potentially important development in coding theory in many years" [16] and have recently been shown [12, 15] to utilize an algorithm equivalent to belief propagation in a network with loops. Although there is widespread agreement in the coding community that these codes "represent a genuine, and perhaps historic, breakthrough" [16] a theoretical understanding of their performance has yet to be achieved.

Progress in the analysis of loopy belief propagation has been made for the case of 
networks with a single loop [22, 23, 6, 2]. For these networks, it can be shown that:

- Unless all the compatibilities are deterministic, loopy belief propagation will converge.

- An analytic expression relates the correct marginals to the loopy marginals. The approximation error is related to the convergence rate of the messages - the faster the convergence the more exact the approximation.

- If the hidden nodes are binary, then the loopy beliefs and the true beliefs are both maximized by the same assignments, although the confidence in that assignment is wrong for the loopy beliefs.

In this paper we analyze belief propagation in graphs of arbitrary topology but focus primarily on nodes that describe jointly Gaussian random variables. We give an exact formula that relates the correct marginal posterior probabilities with the ones calculated using loopy belief propagation. We show that if belief propagation converges then it will give the correct posterior means for all graph topologies, not just networks with a single loop. The covariance estimates will generally be incorrect but we present a relationship between the error in the covariance estimates and the convergence speed. For Gaussian or non-Gaussian variables, we show that the "max-product" algorithm, which calculates the MAP estimate in singly connected networks, only converges to points that are at least local maxima of the posterior probability of loopy networks. This motivates using this powerful algorithm in a broader class of networks.

\section{Belief propagation in undirected graphical models}

An undirected graphical model (or a Markov Random Field) is a graph in which the nodes represent variables and arcs represents compatability constraints between them. Assuming all probabilities are nonzero, the Hammersley-Clifford theorem (e.g. [18]) guarantees that the probability distribution will factorize into a product of functions of the maximal cliques of the graph.

Denoting by $x$ the values of all variables in the graph, the factorization has the form:

$$
P(x)=\prod_{c} \Psi\left(x_{c}\right)
$$

where $x_{c}$ is a subset of $x$ that form a clique in the graph.

We will assume, without loss of generality, that each $x_{i}$ node has a corresponding $y_{i}$ node that is connected only to $x_{i}$.

Thus:

$$
P(x, y)=\prod_{c} \Psi\left(x_{c}\right) \prod_{i} \Psi\left(x_{i}, y_{i}\right)
$$

The restriction that all the $y_{i}$ variables are observed and none of the $x_{i}$ variables are is just to make the notation simple - $\Psi\left(x_{i}, y_{i}\right)$ may be independent of $y_{i}$ (equivalent to $y_{i}$ being unobserved) or $\Psi\left(x_{i}, y_{i}\right)$ may be $\delta\left(x_{i}-x_{o}\right)$ (equivalent to $x_{i}$ being observed, with value $x_{o}$ ). 


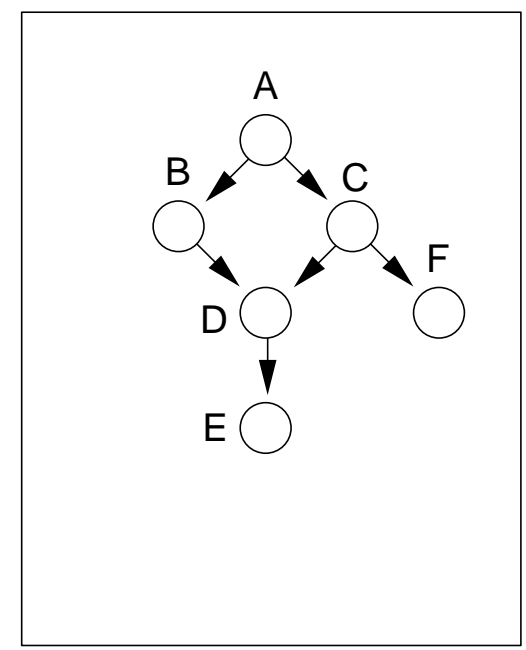

a

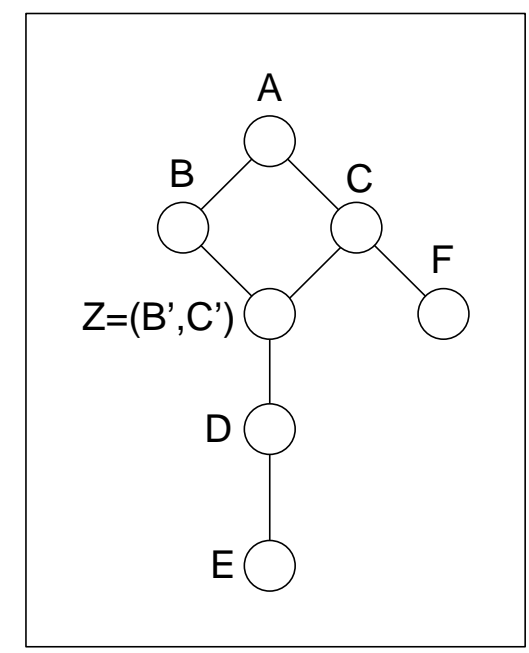

b

Figure 1: Any Bayesian network can be converted into an undirected graph with pairwise cliques by adding cluster nodes for all parents that share a common child. a. A Bayesian network. b. The corresponding undirected graph with pairwise cliques. A cluster node for $(B, C)$ has been added. The potentials can be set so that the joint probability in the undirected network is identical to that in the Bayesian network. In this case the update rules presented in this paper reduce to Pearl's propagation rules in the original Bayesian network [23].

In describing and analyzing belief propagation we assume the graphical model has been preprocessed so that all the maximal cliques consist of pairs of units. Any graphical model can be converted into this form before doing inference through a suitable clustering of nodes into large nodes [23]. Figure 1 shows an example of such a conversion.

Equation 2 becomes

$$
P(x, y)=\prod_{c} \Psi\left(x_{c_{1}}, x_{c_{2}}\right) \prod_{i} \Psi\left(x_{i}, y_{i}\right)
$$

Here each clique $c$ corresponds to an edge in the graph and $x_{c_{1}}, x_{c_{2}}$ refer to the two nodes connected by the edges.

The advantage of preprocessing the graph into one with pairwise cliques is that the description and the analysis of belief propagation becomes simpler. For completeness, we review the belief propagation scheme used in [23].

At every iteration, each node sends a (different) message to each of its neighbors and receives a message from each neighbor. Let $V$ and $W$ be two neighboring nodes in the graph. We denote by $m_{V W}(w)$ the message that node $V$ sends to node $W$. $w$ is the vector-valued random variable at node $W$. We denote by $b_{V}(v)$ the belief at node $V$. 
The belief update (or "sum-product" update) rules are:

$$
\begin{aligned}
m_{V W}(w) & \leftarrow \alpha \int_{v} \Psi(V=v, W=w) \prod_{Z \in N(V) \backslash W} m_{Z V}(v) \\
b_{V}(v) & \leftarrow \alpha \prod_{Z \in N(V)} m_{Z V}(v)
\end{aligned}
$$

where $\alpha$ denotes a normalization constant and $N(V) \backslash W$ means all nodes neighboring $V$, except $W$.

The procedure is initialized with all message vectors set to constant functions. Observed nodes do not receive messages and they always transmit the same vector-if $Y$ is observed to be in state $y$ then $m_{Y X}(x)=\Psi(Y=y, X=x)$. The normalization of $m_{V W}$ in equation 4 is not necessary-whether or not the message are normalized, the belief $b_{V}$ will be identical. However, normalizing the messages avoids numerical underflow and adds to the stability of the algorithm. We assume throughout this paper that all nodes simultaneously update their messages in parallel.

It is easy to show that for singly connected graphs these updates will converge in a number of iterations equal to the diameter of the graph and the beliefs are guaranteed to give the correct posterior marginals: $b_{V}(v)=P(V=v \mid O)$ where $O$ denotes the set of observed variables.

This message passing scheme is equivalent to Pearl's belief propagation in directed graphs of arbitrary clique size - for every message passed in this scheme there exists a corresponding message in Pearl's algorithm when the directed graph is converted to an undirected graph with pairwise cliques [23]. For particular graphs with particular settings of the potentials, Equs. 4-5 yield other well-known Bayesian inference algorithms, such as the forward-backward algorithm in Hidden Markov Models, the Kalman Filter and even the Fast Fourier Transform [1, 12].

A related algorithm, "max-product", changes the integration in equation 4 to a maximization. This message-passing is equivalent to Pearl's "belief revision" algorithm in directed graphs. For particular graphs with particular settings of the potentials, the max-product algorithm is equivalent to the Viterbi algorithm for Hidden Markov Models, and concurrent dynamic programming. We define the max-product assignment at each node to be the value that maximizes its belief (assuming a unique maximizing value exists). For singly connected graphs, the max-product assignment is guaranteed to give the MAP assignment.

\subsection{Gaussian Markov Random Fields}

A Gaussian MRF (GMRF) is an MRF in which the joint distribution is Gaussian. We assume, without loss of generality, that the joint mean is zero (the means can be added-in later), so the joint probability, $P(x)$, is

$$
P(x)=\alpha e^{-\frac{1}{2} x^{T} V_{x x} x}
$$

where $V$ is the inverse covariance matrix and $\alpha$ denotes a normalization constant. The MRF properties guarantee that $V_{x x}(i, j)=0$ if $x_{i}$ is not a neighbor of $x_{j}$. It is straightforward to write the inverse covariance matrix describing the GMRF which respects the statistical dependencies within the graphical model [4]. 
Note that when we expand the term in the exponent we will only get terms of the form $V_{x x}(i, j) x(i) x(j)$. Thus there exist a set of matrices $V_{c}$, one corresponding to each pairwise clique, such that:

$$
P(x)=\alpha \prod_{c} e^{-\frac{1}{2} x_{c}^{T} V_{c} x_{c}}
$$

So that for any pair of nodes $\Psi\left(x_{c}\right)=\alpha e^{-\frac{1}{2} x_{c}^{T} V_{c} x_{c}}$.

\subsection{Inference in Gaussian MRFs}

The joint distribution of $z=\left(\begin{array}{l}x \\ y\end{array}\right)$ is given by:

$$
P(z)=\alpha e^{-\frac{1}{2} z^{T} V z}
$$

where $V$ has the following structure

$$
V=\left(\begin{array}{ll}
V_{x x} & V_{x y} \\
V_{y x} & V_{y y}
\end{array}\right)
$$

Note that because $x_{i}$ is only connected to $y_{i}, V_{x y}$ is zero everywhere except the diagonals.

The marginalization formulas for Gaussians allow us to compute the conditional mean of $x$ given the observations $y$. Writing out the exponent of Eq. 8 and completing the square shows that the mean $\mu$ of $x$, given $y$, is a solution to:

$$
V_{x x} \mu=-V_{x y} y
$$

and the covariance matrix $C_{x \mid y}$ of $x$ given $y$ is:

$$
C_{x \mid y}=V_{x x}^{-1}
$$

We will denote by $C_{x_{i} \mid y}$ the $i$ th row of $C_{x \mid y}$, so the marginal posterior variance of $x_{i}$, given the data, is $C_{x_{i} \mid y}(i)$.

Belief propagation in Gaussian MRFs gives simpler update formulas than the general purpose case (Eqs. 4 and 5). The messages and the beliefs are all Gaussians and the updates can be written directly in terms of the means and covariance matrices. Each node sends and receives a mean vector and covariance matrix to and from each neighbor, in general, each different. The beliefs at a node are calculated by combining the means and covariances of all the incoming messages. For scalar nodes, the beliefs are a weighted average of the incoming messages, inversely weighted by their variance.

We can now state the main question of this paper. What is the relationship between the true posterior means and covariances (calculated using Eq. 10) and the belief propagation means and covariances (calculated using the belief propagation rules Eqs. 4-5)? 

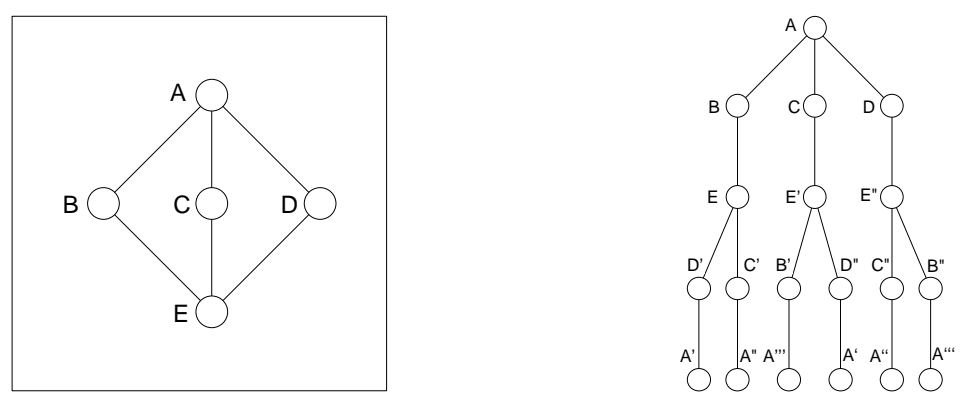

Figure 2: Left: A Markov network with multiple loops. Right: The unwrapped network corresponding to this structure. The unwrapped networks are constructed by replicating the potentials $\Psi\left(x_{i}, x_{j}\right)$ and observations $y_{i}$ while preserving the local connectivity of the loopy network. They are constructed so that the messages received by node $\mathrm{A}$ after $t$ iterations in the loopy network are equivalent to those that would be received by $A$ in the unwrapped network. An observed node, $y_{i}$, not shown, is connected to each depicted node.

\section{Analysis}

To compare the correct posteriors and the loopy beliefs, we construct an unwrapped tree. The unwrapped tree is the graphical model that the loopy belief propagation is solving exactly when applying the belief propagation rules in a loopy network $[9,24,23]$. In error-correcting codes, the unwrapped tree is referred to as the "computation tree" - it is based on the idea that the computation of a message sent by a node at time $t$ depends on messages it received from its neighbors at time $t-1$ and those messages depend on the messages the neighbors received at time $t-2$ etc.

To construct an unwrapped tree, set an arbitrary node, say $x_{1}$, to be the root node and then iterate the following procedure $t$ times:

- Find all leaves of the tree (start with the root).

- For each leaf, find all $k$ nodes in the loopy graph that neighbor the node corresponding to this leaf.

- Add $k-1$ nodes as children to each leaf, corresponding to all neighbors except the parent node.

Each node in the loopy graph will have a different unwrapped tree with that node at the root.

Figure 2 shows an unwrapped tree around node $A$ for the diamond shaped graph on the left. Each node has a shaded observed node attached to it that is not shown for clarity. Since belief propagation is exact for the unwrapped tree, we can calculate the beliefs in the unwrapped tree by using the marginalization formulae for Gaussians.

We use for unwrapped quantities. We scan the tree in breadth first order and denote by $\tilde{x}$ the vector of values in the hidden nodes of the tree when scanned in 
this fashion. Simlarly, we denote by $\tilde{y}$ the observed nodes scanned in the same order. As before, $\tilde{z}=\left(\begin{array}{l}\tilde{x} \\ \tilde{y}\end{array}\right)$ To simplify the notation, we assume throughout this section that all nodes are scalar valued. In section 4 we generalize the analysis to vector valued nodes.

The basic idea behind our analysis is to relate the wrapped and unwrapped inverse covariance matrices. By the nature of unwrapping, all elements $\tilde{V}_{x y}(i, j)$ and $\tilde{y}(i)$ are copies of the corresponding elements $V_{x y}\left(i^{\prime}, j^{\prime}\right)$ and $y\left(i^{\prime}\right)$ (where $\tilde{x}_{i}, \tilde{x}_{j}$ are replicas of $\left.x_{i^{\prime}}, x_{j^{\prime}}\right)$. Also, all elements $\tilde{V}_{x x}(i, j)$ where $i$ and $j$ are non-leaf nodes are copies of $V_{x x}\left(i^{\prime}, j^{\prime}\right)$. However, the elements $\tilde{V}_{x x}(i, j)$ for the leaf nodes are not copies of $V_{x x}\left(i^{\prime}, j^{\prime}\right)$ because leaf nodes are missing some neighbors.

Intuitively, we might expect that if all the equations that $\tilde{\mu}$ satisifies are copies of the equations that $\mu$ satisifies, then simply creating $\tilde{\mu}$ by many copies of $\mu$ would give a valid solution in the unwrapped network. However, because some of the equations are not copies, this intuition does not explain why the means are exact in Gaussian networks.

An additional intuition, that we formalize below, is that the influence of the noncopied equations (those at the leaf nodes) decreases with additional iterations. As the number of iterations is increased, the distance between the leaf nodes and the root node increases and their influence on the root node decreases. When their influence goes to zero, the mean at the root node is exact.

Although the elements $V_{x x}(i, j)$ are copies of $V_{x x}\left(i^{\prime}, j^{\prime}\right)$ for the non-leaf nodes, the matrix $\tilde{V}_{x x}$ is not simply a block replication of $V_{x x}$. The system of equations that defines $\tilde{\mu}$ is a coupled system of equations. Hence the variance at the root node $\tilde{V}_{x x}^{-1}(1,1)$ differs from the correct variance $V_{x x}^{-1}(1,1)$.

In the following section we prove the following three claims.

Assume, without loss of generality, that the root node is $x_{1}$. Let $\tilde{\mu}(1)$ and $\tilde{\sigma}^{2}(1)$ be the conditional mean and variance at node 1 after $t$ iterations of loopy propagation. Let $\mu(1)$ and $\sigma^{2}(1)$ be the correct conditional mean and variance of node 1 . Let $\tilde{C}_{x_{1} \mid y}$ be the conditional correlation of the root node with all other nodes in the unwrapped tree then:

\section{Claim 1:}

$$
\tilde{\mu}(1)=\mu(1)+\tilde{C}_{x_{1} \mid y} r
$$

where $r$ is a vector that is zero everywhere but the last $L$ components (corresponding to the leaf nodes).

\section{Claim 2:}

$$
\tilde{\sigma}^{2}(1)=\sigma^{2}(1)+\tilde{C}_{x_{1} \mid y} r_{1}-\tilde{C}_{x_{1} \mid y} r_{2}
$$

where $r_{1}$ is a vector that is zero everywhere but the last $L$ components and $r_{2}$ is equal to 1 for all components corresponding to non-root nodes in the unwrapped tree that reference $x_{1}$. All other components of $r_{2}$ are zero.

Claim 3: If the conditional correlation between the root node and the leaf nodes decreases rapidly enough then (1) belief propagation converges (2) the belief propagation means are exact and (3) the belief propagation variances are equal to the correct variances minus the summed conditional correlations between $\tilde{x}_{1}$ and all $\tilde{x}_{j}$ 


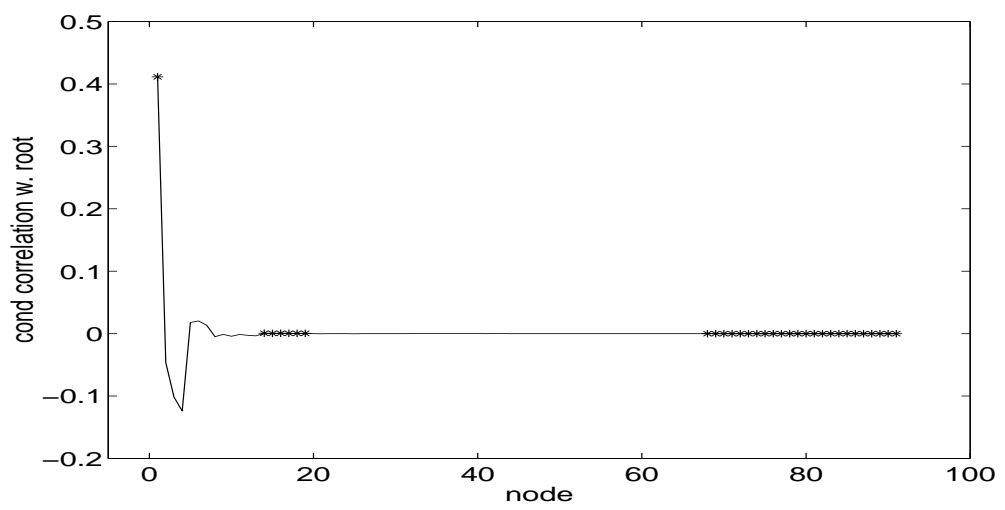

Figure 3: The conditional correlation between the root node and all other nodes in the unwrapped tree for the diamond figure after 15 iterations. Potentials were chosen randomly. Nodes are presented in breadth first order so the last elements are the correlations between the root node and the leaf nodes. It can be proven that if this correlation goes to zero then (1) belief propagation converges (2) the loopy means are exact and (3) the loopy variances equal the correct variances minus the summed conditional correlation of the root node and all other nodes that are replicas of the same loopy node. Symbols plotted with a star denote correlations with nodes that correspond to the node $A$ in the loopy graph. It can be proven that the sum of these correlations gives the correct variance of node $A$ while loopy propagation uses only the first correlation.

that are replicas of $x_{1}$.

To obtain intuition, Fig. 3 shows $\tilde{C}_{x_{1} \mid y}$ for the diamond figure in Fig. 2. We generated random potential functions and observations for the loopy diamond figure and calculated the conditional correlations in the unwrapped network. Note that the conditional correlation decreases with distance in the tree - we are scanning in breadth first order so the last $L$ components correspond to the leaf nodes. As the number of iterations of loopy propagation is increased the size of the unwrapped tree increases and the conditional correlation between the leaf nodes and the root node decreases.

From equations $12-13$ it is clear that if the conditional correlation between the leaf nodes and the root nodes are zero for all sufficiently large unwrappings then (1) belief propagation converges (2) the means are exact and (3) the belief propagation variances are equal to the correct variances minus the summed conditional correlations between $\tilde{x}_{1}$ and all $\tilde{x}_{j}$ that are replicas of $x_{1}$. In practice the conditional correlations will not actually be equal to zero for any finite unwrapping so claim 3 states this more precisely.

\subsection{Relation of loopy and unwrapped quantities}

The proof of all three claims relies on the relationship between the elements of $y, V_{x y}$ and $V_{x x}$ with their unwrapped quantities, described below.

Each node in $\tilde{x}$ corresponds to a node in the original loopy network. Let $O$ be a 
matrix that defines this correspondence. $O(i, j)=1$ if $\tilde{x}_{i}$ corresponds to $x_{j}$ and zero otherwise. Thus, in figure 2, ordering the nodes alphabetically, the first rows of $O$ are:

$$
O=\left(\begin{array}{ccccc}
1 & 0 & 0 & 0 & 0 \\
0 & 1 & 0 & 0 & 0 \\
0 & 0 & 1 & 0 & 0 \\
0 & 0 & 0 & 1 & 0 \\
0 & 0 & 0 & 0 & 1 \\
0 & 0 & 0 & 0 & 1 \\
0 & 0 & 0 & 0 & 1 \\
0 & 0 & 0 & 1 & 0 \\
0 & 0 & 1 & 0 & 0 \\
0 & 1 & 0 & 0 & 0 \\
\ldots & \ldots & \ldots & \ldots & \ldots
\end{array}\right)
$$

Using $O$ we can formalize the relationship between the unwrapped quantities and the original ones. The simplest one is $\tilde{y}$, that only contains replicas of the original $y$ :

$$
\tilde{y}=O y
$$

Since every $x_{i}$ is connected to a $y_{i}, V_{x y}$ and $\tilde{V}_{x y}$ are zero everywhere but along their diagonals (the block diagonals, for vector valued variables). The diagonal elements of $\tilde{V}_{x y}$ are simply replications of $V_{x y}$ hence:

$$
\tilde{V}_{x y} O=O V_{x y}
$$

$\tilde{V}_{x x}$ also contains the elements of the original $V_{x x}$ but here special care needs to be taken. Note that by construction, every node in the interior of the unwrapped tree has exactly the same statistical relationship with its neighbors as with the corresponding node in the loopy graph. If a node in the loopy graph has $k$ neighbors then a node in the unwrapped tree will have, by construction, one parent and $k-1$ children. The leaf nodes in the unwrapped tree, however, will be missing the $k-1$ children and hence will not have the same number of neighbors. Thus, for all nodes $\tilde{x}_{i}, \tilde{x}_{j}$ that are not leaf nodes, $\tilde{V}_{x x}(i, j)$ is a copy of the corresponding $V_{x x}(k, l)$, where unwrapped nodes $i$ and $j$ refer to loopy nodes $k$ and $l$, respectively.

Therefore:

$$
\tilde{V}_{x x} O+E=O V_{x x}
$$

where $E$ is an error matrix. E is zero for all non-leaf nodes so the first $N-L$ rows of $\mathrm{E}$ are zero.

\subsection{Proof of claim 1}

The marginalization equation for the unwrapped problem gives:

$$
\tilde{V}_{x x} \tilde{\mu}=-\tilde{V}_{x y} \tilde{y}
$$

Substituting Eqs. 15 and 16, relating loopy and unwrapped network quantities, into Eq. 18, for the unwrapped posterior mean, gives:

$$
\tilde{V}_{x x} \tilde{\mu}=-O V_{x y} y
$$


For the true means, $\mu$, of the loopy network, we have

$$
V_{x x} \mu=-V_{x y} y
$$

To relate that to the means of the unwrapped network, we left-multiply by $O$ :

$$
O V_{x x} \mu=-O V_{x y} y .
$$

Using Eq. 17, relating $V_{x x}$ to $\tilde{V}_{x x}$, we have

$$
\tilde{V}_{x x} O \mu+E \mu=-O V_{x y} y
$$

Comparing Eqs. 22 and 19 gives

$$
\tilde{V}_{x x} O \mu+E \mu=\tilde{V}_{x x} \tilde{\mu}
$$

or:

$$
\tilde{\mu}=O \mu+\tilde{V}_{x x}^{-1} E \mu
$$

Using Eq. 11

$$
\tilde{\mu}=O \mu+\tilde{C}_{x \mid y} E \mu
$$

The left and right hand sides of equation 25 are column vectors. We take the first component of both sides and get:

$$
\tilde{\mu}(1)=\mu(1)+\tilde{C}_{x_{1} \mid y} E \mu
$$

Since $E$ is zero in the first $N-L$ rows, $E \mu$ is zero in the first $N-L$ components.

\subsection{Proof of claim 2}

From Eq. 11,

$$
V_{x x} C_{x \mid y}=I
$$

Taking the first column of this equation gives:

$$
V_{x x} C_{x_{1} \mid y}^{T}=e_{1}
$$

where $e_{1}(1)=1, e_{1}(j>1)=0$.

Using the same strategy as in the previous proof, we left multiply by $O$ :

$$
O V_{x x} C_{x_{1} \mid y}^{T}=O e_{1}
$$

and similarly we substitute equation 17 :

$$
\tilde{V}_{x x} O C_{x_{1} \mid y}^{T}+E C_{x_{1} \mid y}^{T}=O e_{1}
$$

The analog of equation 28 in the unwrapped problem is:

$$
\tilde{V}_{x x} \tilde{C}_{x_{1} \mid y}^{T}=\tilde{e}_{1}
$$


where $\tilde{e}_{1}(1)=1, \tilde{e}_{1}(j>1)=0$.

Subtracting Eqs. 30 and 31 and rearranging terms gives:

$$
\tilde{C}_{x_{1} \mid y}=O C_{x_{1} \mid y}^{T}+\tilde{V}_{x x}^{-1} E C_{x_{1} \mid y}^{T}+\tilde{V}_{x x}^{-1}\left(\tilde{e}_{1}-O e_{1}\right)
$$

Again, we take the first row of both sides of equation 32 and use the fact that the first row of $\tilde{V}_{x x}^{-1}$ is $\tilde{C}_{x_{1} \mid y}$ to obtain:

$$
\tilde{\sigma}^{2}(1)=\sigma^{2}(1)+\tilde{C}_{x_{1} \mid y} E C_{x_{1} \mid y}^{T}+\tilde{C}_{x_{1} \mid y}\left(\tilde{e}_{1}-O e_{1}\right)
$$

Again, since $E$ is zero in the first $N_{L}$ rows, $E C_{x_{1} \mid y}$ is zero in the first $N-L$ components.

\subsection{Proof of claim 3}

Here we need to define what we mean by "rapidly enough". We restate the claim precisely.

Suppose for every $\epsilon$ there exists a $t_{\epsilon}$ such that for all $t>t_{\epsilon}\left|\tilde{C}_{x_{1} \mid y} r\right|<\epsilon \max _{i}|r(i)|$ for any vector $r$ that is nonzero only in the last $L$ components (those corresponding to the leaf nodes). In this case, (1) belief propagation converges (2) the means are exact and (3) the variances are equal to the correct variances minus the summed conditional correlations between $\tilde{x}_{1}$ and all non-root $\tilde{x}_{j}$ that are replicas of $x_{1}$

This claim follows from the first two claims. The only thing to show is that $E \mu$ and $E C_{x_{1} \mid y}$ are bounded for all iterations. This is true because the rows of $E$ are bounded and $\mu, C_{x_{1} \mid y}$ do not depend on the iteration.

How wrong will the variances be? The term $\tilde{C}_{x_{1} \mid y} r_{2}$ in Eq. 13 is simply the sum of many components of $\tilde{C}_{x_{1} \mid y}$. Figure 3 shows these components. The correct variance is the sum of all the components while the loopy variance approximates this sum with the first (and dominant) term.

Note that when the conditional correlation decreases rapidly to zero two things happen. First, the convergence is faster (because $\tilde{C}_{x_{1} \mid y} r_{1}$ approaches zero faster). Second, the approximation error of the variances is smaller (because $\tilde{C}_{x_{1} \mid y} r_{2}$ is smaller). Thus, as in the single loop case, we find that quick convergence is correlated with good approximation.

In practice, it may be difficult to check whether the conditional correlations decrease rapidly enough. In the next section we show that if loopy propagation converges then the loopy means are exact.

\section{$3 \quad$ Fixed points of loopy propagation}

Each iteration of belief propagation can be thought of as an operator $F$ that inputs a list of messages $m^{(t)}$ and outputs a list of messages $m^{(t+1)}=F m^{(t)}$. Thus belief propagation can be thought of as an iterative way of finding a solution to the fixed point equations $F m=m$ with an initial guess $m_{0}$ in which all messages are constant functions. 
Note that this is not the only way of finding fixed-points. McEliece et al. [16] have shown a simple example for which $F$ contains multiple fixed points and belief propagation finds only one. They also showed a simple example where a fixed-point exists but the iterations $m=F m$ do not converge. An alternative way of finding fixed-points of $F$ is described in [17].

In this section we ask, suppose a fixed-point $m^{*}=F m^{*}$ has been found by some method, how are the beliefs calculated based on these messages related to the correct beliefs?

Claim 4: For a Gaussian graphical model of arbitrary topology, if $m^{*}$ is a fixedpoint of the message-passing dynamics then the means based on that fixed-point are exact.

The proof is based on the following lemma:

Periodic beliefs lemma: If $m^{*}$ is a fixed-point of the message-passing dynamics in a graphical model $G$ then one can construct a modified unwrapped tree $T$ of arbitrarily large depth such that: (1) all non-leaf nodes in $T$ have the same statistical relationship with their neighbors as the corresponding nodes in $G$ and (2) all nodes in $T$ will have the same belief as the beliefs in $G$ derived from $m^{*}$.

Proof: The proof is by construction. We first construct an unwrapped tree $T$ of the desired depth. We then modify the potentials and the observations in the leaf nodes in the following manner. For each leaf node $\tilde{x}_{i}$, find the $k-1$ nodes in $G$ that neighbor $x_{i^{\prime}}$ (where $\tilde{x}_{i}$ is a replica of $x_{i^{\prime}}$ ) excluding the parent of $\tilde{x}_{i}$. Calculate the product of the $k-1$ messages that these neighbors send to the corresponding node in $G$ under the fixed-point messages $m^{*}$ and the message that $y_{i^{\prime}}$ sends to $x_{i^{\prime}}$. Set $\tilde{y}_{i}$ and $\Psi\left(\tilde{y}_{i}, \tilde{x}_{i}\right)$ such that the message $\tilde{y}_{i}$ sends to $\tilde{x}_{i}$ is equal to this product.

By this construction, all leaf nodes in $T$ will send their neighbors a message from $m^{*}$. Since all non-leaf nodes in $T$ have the same statistical relationship to their neighbors as the corresponding nodes in $G$, the local message passing updates in $T$ are identical to those in $G$. Thus all messages in $T$ will be replicas of messages in $m^{*}$.

Proof of Claim 4: Using this lemma we can prove claim 4. Let $\tilde{\mu}$ be the conditional mean in the modified unwrapped tree then, by the periodic beliefs lemma:

$$
\tilde{\mu}=O \mu_{0}
$$

where $\mu_{0}(i)$ is the posterior mean at node $i$ under $m^{*}$.

We also know that $\tilde{\mu}$ is a solution to:

$$
\tilde{V}_{x x} \tilde{\mu}=-\tilde{V}_{x y} \tilde{y}
$$

where $\tilde{V}_{x x}, \tilde{V}_{x y}, \tilde{y}$ refers to quantities in the modified unwrapped tree. So:

$$
\tilde{V}_{x x} O \mu_{0}=-\tilde{V}_{x y} \tilde{y}
$$

We use the notation $[A]_{m}$ to indicate taking the $m$ first rows of a matrix $A$. Note that for any two matrices $[A B]_{m}=[A]_{m} B$. Taking the first $m$ rows of equation 36 gives:

$$
\left[\tilde{V}_{x x} O\right]_{m} \mu_{0}=-\left[\tilde{V}_{x y} \tilde{y}\right]_{m}
$$


As in the previous proofs, the key idea is to relate the inverse covariance matrix of the modified unwrapped tree to that of the original loopy graph. Since all non-leaf nodes in the modified unwrapped tree have the same neighborhood relationships with their neighbors as the corresponding nodes in the loopy graph we have, for any $m<N-L$ :

$$
\left[\tilde{V}_{x x} O\right]_{m}=\left[O V_{x x}\right]_{m}
$$

and:

$$
\left[\tilde{V}_{x y} \tilde{y}\right]_{m}=\left[O V_{x y} y\right]_{m}
$$

Substituting these relationships into equation 37 gives:

$$
[O]_{m} V_{x x} \mu_{0}=-[O]_{m} V_{x y} y
$$

This equation holds for any $m<N-L$. Since we can unwrap the tree to arbitrarily large size we can choose $m$ such that $[O]_{m}$ has $n$ independent rows (this happens once all nodes in the loopy graph appear at least once in the modified unwrapped tree). Thus:

$$
V_{x x} \mu_{0}=-V_{x y} y
$$

hence the means derived from the fixed-point messages are exact.

\subsection{Non-Gaussian variables}

In Sect. 1 we described the "max-product" belief propagation algorithm that finds the MAP estimate for each node $[18,23]$ of a network without loops. As with maxproduct, iterating this algorithm is a method of finding a fixed-point of the message passing dynamics. How does the assignment derived from this fixed-point compare the MAP assignment?

Claim 5: For a graphical model of arbitrary toplogy with continuous potential functions, if $m^{*}$ is a fixed-point of the max-product message-passing dynamics then the assignment based on that fixed-point is a local maximum of the posterior probability.

Since the posterior probability factorizes into a product of pairwise potentials, the $\log$ posterior will have the form,

$$
\log P(x \mid y)=\sum_{i j} J_{i j}\left(x_{i}, x_{j}\right)+J_{i i}\left(x_{i}, y_{i}\right)
$$

Assuming the clique potential functions are differentiable and finite, the MAP solution, $u$, will satisfy

$$
\left.\frac{\partial}{\partial x_{i}} \log P(x \mid y)\right|_{x=u}=0
$$

We will write this as:

$$
V u=0
$$

where $V$ is a nonlinear operator.

As in the previous section, we can use the periodic belief lemma to construct a modified unwrapped tree of arbitrary size based on $m^{*}$. If we denote by $\tilde{V}$ the 
nonlinear set of equations that the solution to the modified unwrapped problem must satisfy we have:

$$
\tilde{V} \tilde{u}=0
$$

Because of the periodic belief lemma:

$$
\tilde{u}=O u_{0}
$$

Similarly, as in the previous section, all the non-leaf nodes will have the same statistical relationship with their neighbors as do the corresponding nodes in the loopy network, so:

$$
[\tilde{V} O]_{m}=[O V]_{m}
$$

where the left and right hand sides are nonlinear operators.

Substituting Eqs. 46 and 47 into Eq. 45 gives:

$$
V u_{0}=0
$$

A similar substitution can be made with the second derivative equations to show that the Hessian at $u_{0}$ is positive definite. Thus the assignment based on $m^{*}$ is at least a local maximum of the posterior.

\section{Vector valued nodes}

All of the results we have derived so far hold for vector-valued nodes as well but the indexing notation is slightly more cumbersome. We use a stacking convention, in which we define the vector $x$ by:

$$
x=\left(\begin{array}{c}
x_{1} \\
x_{2} \\
\cdots
\end{array}\right)
$$

Thus supposing $x_{1}$ is a vector of length 2 then $x(1)$ is the first component of $x_{1}$ and $x(2)$ is the second component of $x_{1}\left(\right.$ not $\left.x_{2}\right)$. We define $y$ in a similar fashion.

Using this stacking notation the equations for exact inference in Gaussians remain unchanged, but we need to be careful in reading out the posterior means and covariances from the stacked vectors. Thus we can still complete the square in stacked notation to obtain:

$$
V_{x x} \mu=-V_{x y} y
$$

and $C_{x \mid y}=V_{x x}^{-1}$. Assuming $x_{1}$ is of length 2, $\mu_{1}$ the posterior mean of $x_{1}$ is given by:

$$
\mu_{1}=\left(\begin{array}{l}
\mu(1) \\
\mu(2)
\end{array}\right)
$$

and the posterior covariance matrix $\Sigma_{1}$ is given by:

$$
\Sigma_{1}=\left(\begin{array}{cc}
C_{x \mid y}(1,1) & C_{x \mid y}(1,2) \\
C_{x \mid y}(2,1) & C_{x \mid y}(2,2)
\end{array}\right)
$$


We use the same stacked notation for $\tilde{x}$ and define the matrix $O$ such that $O(i, j)=1$ if $\tilde{x}(i)$ is a replica of $x(j)$ and zero otherwise. Using this notation, the relationships between unwrapped and loopy quantities (e.g. $\left[\tilde{V}_{x x} O\right]_{m}=\left[O V_{x x}\right]_{m}$ ) still hold. Thus all the analysis done in the previous sections holds - the only difference are the semantics of quantities such as $\mu(1)$, which need to be understood as a scalar component of a (possibly) larger vector $\mu_{1}$. For explicitness, we restate the five claims for vector valued nodes.

For any $i, j$ less than or equal to the number of components in $x_{1}$ we have:

\section{Claim 1a:}

$$
\tilde{\mu}(i)=\mu(i)+\tilde{C}_{x_{i} \mid y} r
$$

where $r$ is a vector that is zero everywhere but the last $L$ components (corresponding to the leaf nodes).

\section{Claim 2a:}

$$
\tilde{C}_{x \mid y}(i, j)=C_{x \mid y}(i, j)+\tilde{C}_{x_{j} \mid y} r_{1}-\tilde{C}_{x_{j} \mid y} r_{2}
$$

where $r_{1}$ is a vector that is zero everywhere but the last $L$ components (corresponding to the leaf nodes) and $r_{2}$ is equal to 1 for all components corresponding to non-root nodes in the unwrapped tree that reference $x(i)$. All other components of $r_{2}$ are zero.

Claim 3a: If the conditional correlation between all components of the root node and the leaf nodes decreases rapidly enough then (1) belief propagation converges (2) the belief propagation means are exact and (3) the $i, j$ component of the belief propagation covariance matrices is equal to the $i, j$ component of the true covariance matrices minus the summed conditional correlations between $\tilde{x}(j)$ and all nonroot $\tilde{x}(k)$ that are replicas of $x(i)$.

Claim 4a: For a (possibly vector-valued) Gaussian graphical model of arbitrary topology, if $m^{*}$ is a fixed-point of the message-passing dynamics, then the means based on that fixed-point are exact.

Claim 5a: For a (possibly vector-valued) graphical model of arbitrary topology with continuous potential functions, if $m^{*}$ is a fixed-point of the max-product message-passing dynamics, then the assignment based on that fixed-point is a local maximum of the posterior probability.

We emphasize that these claims do not need to be reproved - all the equations used in proving the scalar-valued case still hold only the semantics we place on the individual components are different.

We end this analysis with two simple corollaries:

Corollary 1: Let $m^{*}$ be a fixed-point of Pearl's belief propagation algorithm on a Gaussian Bayesian network of arbitrary toplogy and arbitrary clique size. Then the means based on $m^{*}$ are exact.

Corollary 2: Let $m^{*}$ be a fixed-point of Pearl's belief revision (max-product) algorithm on a Bayesian network with continuous joint probability, arbitrary topology and arbitrary clique size. The assignment based on $m^{*}$ is at least a local maximum of the posterior probability.

These corollaries follow from claims 4 a and 5 a along with the equivalence between 


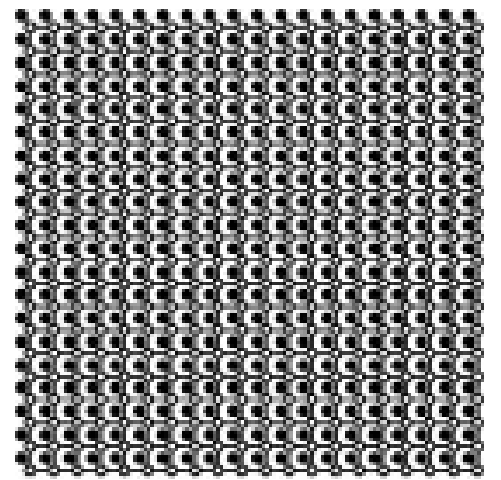

(a)

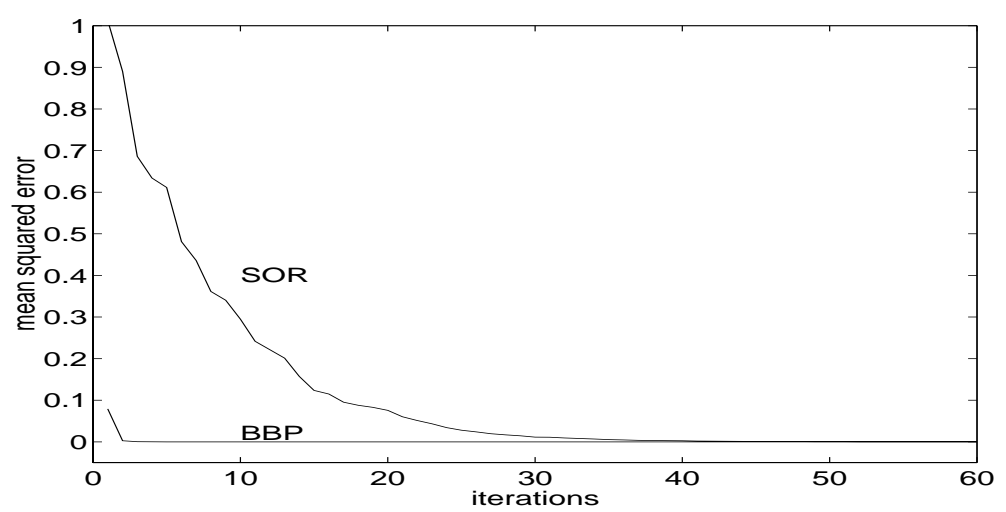

(b)

Figure 4: (a) $25 \times 25$ graphical model for simulation. The unobserved nodes (unfilled) were connected to their four nearest neighbors and to an observation node (filled). (b) The error of the estimates of loopy propagation and successive overrelaxation (SOR) as a function of iteration. Note that belief propagation converges much faster than SOR.

Pearl's propagation rules and the propagation rules for pairwise undirected graphical models analyzed here [23]. Note that even if the Bayesian network contained only scalar nodes, the conversion to pairwise cliques may necessitate using vector-valued nodes.

\section{Simulations}

We ran belief propagation on a $25 \times 252 \mathrm{D}$ grid. The joint probability was:

$$
P(x, y)=\exp \left(-\sum_{i j} w_{i j}\left(x_{i}-x_{j}\right)^{2}-\sum_{i} w_{i i}\left(x_{i}-y_{i}\right)^{2}\right)
$$

where $w_{i j}=0$ if nodes $x_{i}, x_{j}$ are not neighbors and 0.01 otherwise and $w_{i i}$ was randomly selected to be 0 or 1 for all $i$ with probability of 1 set to 0.2 . The observations $y_{i}$ were chosen randomly. This problem corresponds to an approximation problem from sparse data where only $20 \%$ of the points are visible.

We found the exact posterior by solving Eq. 10. We also ran loopy belief propagation and found that when it converged, the loopy means were identical to the true means up to machine precision. Also, as explained by the theory, the loopy variances were too small - the loopy estimate was overconfident.

In many applications, the solution of equation 10 by matrix inversion is intractable and iterative methods are used. Figure 4 compares the error in the means as a function of iterations for loopy propagation and successive-over-relaxation (SOR), considered one of the best relaxation methods [20]. Note that after five iterations loopy propagation gives essentially the right answer while SOR requires many more. As expected by the fast convergence, the approximation error in the variances was quite small. The median error was 0.018 . For comparison the true variances ranged from 0.01 to 0.94 with a mean of 0.322 . Also, the nodes for which the approximation error was worse were indeed the nodes that converged slower. 
The slow convergence of SOR on problems such as these lead to the development of multi-resolution models in which the MRF is approximated by a tree [14, 5] and an algorithm equivalent to belief propagation is then run on the tree. Although the multi-resolution models are much more efficient for inference, the tree structure often introduces block artifacts in the estimate. Our results suggest that one can simply run belief propagation on the original MRF and get the exact posterior means. Although the posterior variances will not be correct, for those nodes for which loopy propagation converged rapidly the approximation error will be small.

\section{Discussion}

Our main interest in analyzing the Gaussian case was to understand the performance of belief propagation in networks with multiple loops. Although there are many special properties of Gaussians, we are struck by the similarity of the analytical results reported here for multi-loop Gaussians and the analytical results for single loops and general distributions reported in [23]. The most salient similarities are:

- In single loop networks with binary nodes, the mode at each node is guaranteed to be correct but the confidence in the mode may be incorrect. In Gaussian networks with multiple loops the mean at each node is guaranteed to be correct but the confidence around that mean will in general be incorrect.

- In single loop networks fast convergence is correlated with good approximation of the beliefs. This is also true for Gaussian networks with multiple loops.

- In single loop networks the convergence rate and the approximation error were determined by a ratio of eigenvalues $\lambda_{1} / \lambda_{2}$. This ratio determines the extent of the statistical dependencies between the root and the leaf nodes in the unwrapped network for a single loop. In Gaussian networks the convergence rate and the approximation error are determined by the off-diagonal terms of $\tilde{C}_{x \mid y}$. These terms quantify the extent of conditional dependencies between the root nodes and the leaf nodes of the unwrapped network.

These similarities are even more intriguing when one considers how different Gaussians graphical models are from discrete models with arbitrary potentials and a single loop. In Gaussians the conditional mean is equal to the conditional mode and there is only one maximum in the posterior probability, while the single loop discrete models may have multiple maxima, none of which will be equal to the mean. Furthermore, in terms of approximate inference the two classes behave quite differently. For example, mean field approximations are exact for Gaussian MRFs while they work poorly in discrete networks with a single loop in which the connectivity is sparse [21]. The resemblance of the results for Gaussian graphical models and for single loops leads us to believe that similar results may hold for a larger class of networks.

The sum-product and max-product belief propagation algorithms are appealing, fast and easily parallelizable algorithms. Due to the well known hardness of probabilistic inference in graphical models, belief propagation will obviously not work 
for arbitrary networks and distributions. Nevertheless, there is a growing body of empirical evidence showing its success in many loopy networks. Our results give a theoretical justification for applying belief propagation in networks with multiple loops. This may enable fast, approximate probabilistic inference in a range of new applications.

\section{Acknowledgments}

We thank A. Ng, K. Murphy, P. Pakzad and M.I. Jordan for comments on previous versions of this manuscript.

\section{References}

[1] S. M. Aji and R.J. McEliece. The generalized distributive law. submitted to IEEE Transactions on Information Theory, 1998.

[2] S.M. Aji, G.B. Horn, and R.J. McEliece. On the convergence of iterative decoding on graphs with a single cycle. In Proc. 1998 ISIT, 1998.

[3] C. Berrou, A. Glavieux, and P. Thitimajshima. Near Shannon limit errorcorrecting coding and decoding: Turbo codes. In Proc. IEEE International Communications Conference '93, 1993.

[4] R. Cowell. Advanced inference in Bayesian networks. In M.I. Jordan, editor, Learning in Graphical Models. MIT Press, 1998.

[5] M. M. Daniel and A. S. Willsky. The modeling and estimation of statistically self-similar processes in a multiresolution framework. IEEE Trans. Info. Theory, 45(3):955-970, April 1999.

[6] G.D. Forney, F.R. Kschischang, and B. Marcus. Iterative decoding of tailbiting trellisses. preprint presented at 1998 Information Theory Workshop in San Diego, 1998.

[7] W.T. Freeman and E.C. Pasztor. Learning to estimate scenes from images. In M.S. Kearns, S.A. Solla, and D.A. Cohn, editors, Adv. Neural Information Processing Systems 11. MIT Press, 1999.

[8] Brendan J. Frey. Bayesian Networks for Pattern Classification, Data Compression and Channel Coding. MIT Press, 1998.

[9] R.G. Gallager. Low Density Parity Check Codes. MIT Press, 1963.

[10] S. Geman and D. Geman. Stochastic relaxation, Gibbs distributions, and the Bayesian restoration of images. IEEE Trans. PAMI, 6(6):721-741, November 1984.

[11] F.V. Jensen. An Introduction to Bayesian Networks. Springer, 1996.

[12] F. R. Kschischang and B. J. Frey. Iterative decoding of compound codes by probability propagation in graphical models. IEEE Journal on Selected Areas in Communication, 16(2):219-230, 1998.

[13] S. Lauritzen. Graphical Models. Oxford University Press, 1996. 
[14] Mark R. Luettgen, W. Clem Karl, and Allan S. Willsky. Efficient multiscale regularization with application to the computation of optical flow. IEEE Transactions on image processing, 3(1):41-64, 1994.

[15] R.J. McEliece, D.J.C. MackKay, and J.F. Cheng. Turbo decoding as as an instance of Pearl's 'belief propagation' algorithm. IEEE Journal on Selected Areas in Communication, 16(2):140-152, 1998.

[16] R.J. McEliece, E. Rodemich, and J.F. Cheng. The Turbo decision algorithm. In Proc. 33rd Allerton Conference on Communications, Control and Computing, pages 366-379, Monticello, IL, 1995.

[17] K.P. Murphy, Y. Weiss, and M.I. Jordan. Loopy belief propagation for approximate inference: an empirical study. In Proceedings of Uncertainty in AI, 1999.

[18] Judea Pearl. Probabilistic Reasoning in Intelligent Systems: Networks of Plausible Inference. Morgan Kaufmann, 1988.

[19] P. Smyth. Belief networks, hidden Markov models, and Markov random fields: a unifying view. Pattern Recognition, 18(11):1261-1268, 1997.

[20] Gilbert Strang. Introduction to Applied Mathematics. Wellesley-Cambridge, 1986.

[21] Y. Weiss. Interpreting images by propagating Bayesian beliefs. In M. Mozer, M. Jordan, and T. Petsche, editors, Advances in Neural Information Processing Systems 9, 1996.

[22] Y. Weiss. Belief propagation and revision in networks with loops. Technical Report 1616, MIT AI lab, 1997.

[23] Y. Weiss. Correctness of local probability propagation in graphical models with loops. Neural Computation, to appear, 1999.

[24] N. Wiberg. Codes and decoding on general graphs. PhD thesis, Department of Electrical Engineering, U. Linkoping, Sweden, 1996. 